\title{
Accuracy and applicability of the revised WHO classification (2009) of dengue
}

\author{
V. Wiwanitkit
}

Received: 12 February 2013/Accepted: 15 February 2013/Published online: 9 March 2013

(C) Springer-Verlag Berlin Heidelberg 2013

Sir, the recent report on "Accuracy and applicability of the revised WHO classification (2009) of dengue" is very interesting [1]. Prasad et al. [1] concluded that "The revised WHO/TDR (2009) classification has very high sensitivity for identifying severe dengue and is easy to apply". Indeed, the classification is exactly useful for grouping of the patient for further management [2]. However, whether the classification is useful for predicting of severity and outcome is still controversial. Indeed, any clinical presentations as well as the important laboratory findings (especially for platelet count and hematocrit) are reported for be useless predictors [3]. A recent report by Premaratna et al. [4] also showed that the present recommended predictors of dengue were limited. In patients who received fluid replacement therapy, the closed observation to monitor the clinical change is still needed [5]. Finally, although the new classification can be applicable, this does not mean the classification is easy. There are many parameters to be collected and this might take time. In some cases, urgent management might be required rather than waiting for the complete classification.
Conflict of interest None.

\section{References}

1. Prasad D, Kumar C, Jain A, Kumar R. Accuracy and applicability of the revised WHO classification (2009) of dengue in children seen at a tertiary healthcare facility in northern India. Infection. 2013. (Epub ahead of print).

2. Lin CY, Huang $\mathrm{CH}$, Chen YH. Classification of dengue: the clinical use of World Health Organization 2009 guideline. J Formos Med Assoc. 2013;112(2):61-3.

3. Wiwanitkit V, Manusvanich P. Can hematocrit and platelet determination on admission predict shock in hospitalized children with dengue hemorrhagic fever? A clinical observation from a small outbreak. Clin Appl Thromb Hemost. 2004;10(1):65-7.

4. Premaratna R, Ragupathy A, Miththinda JK, de Silva HJ. Timing, predictors, and progress of third space fluid accumulation during preliminary phase fluid resuscitation in adult patients with dengue. Int J Infect Dis. 2013. doi:10.1016/j.ijid.2012.12.021. (Epub ahead of print).

5. Wiwanitkit V. Dengue fever: diagnosis and treatment. Expert Rev Anti Infect Ther. 2010;8(7):841-5.

\footnotetext{
V. Wiwanitkit

Hainan Medical University, Haikou, China

V. Wiwanitkit

Faculty of Medicine, University of Nis, Nis, Serbia

V. Wiwanitkit ( $\square$ )

Wiwanitkit House, Bangkhae, Bangkok, Thailand

e-mail:wviroj@yahoo.com
} 